\title{
Properties of the jet production in pp collisions measured with the ATLAS detector
}

\author{
Alexander Solodkov* on behalf of the ATLAS collaboration \\ State research center Institute for High Energy Physics, 142281, Protvino, Russia \\ E-mail: Sanya.Solodkov@cern.ch
}

Several aspects of the jet production in $p p$ collisions at $7 \mathrm{TeV}$ have been investigated by the ATLAS collaboration using data recorded in 2010:

- Six possible combinations of light-flavour, charm and bottom jets are identified in dijet events, where the jet flavour is defined by the presence of bottom, charm or solely light flavour hadrons in the jet. Jet flavour composition as well as the ratio in bottom jet production rates in leading and subleading jets have been measured.

- A measurement of the inclusive jet multiplicity ratio is sensitive to the strong coupling constant $\alpha_{S}$ and has reduced sensitivity to the uncertainties due to parton distribution functions. The ATLAS data have been used to determine the strong coupling strength at the $\mathrm{TeV}$ energy scale.

- A measurement of splitting scales in the $k_{\mathrm{T}}$ clustering algorithm using jets from $W+$ jets events provides a way to investigate jet clustering at different resolution scales.

These measurements constitute precision tests of QCD in a new energy regime. Overall a good agreement with QCD expectations is observed. Obtained results can be used for further fine tuning of various event generators.

The European Physical Society Conference on High Energy Physics -EPS-HEP2013

18-24 July 2013

Stockholm, Sweden

${ }^{*}$ Speaker. 


\section{Introduction}

This contribution describes the main results of three selected jet measurements performed using the ATLAS detector [1] at the LHC [2]. The first two analyses are based on the full 2010 data sample corresponding to an integrated luminosity of $39 \mathrm{pb}^{-1}$ and the third one uses $36 \mathrm{pb}^{-1}$ of 2010 data. Further details of the measurements can be found elsewhere [3], [4], [5].

\section{Jet flavour composition}

The production of jets containing bottom and charm hadrons is less affected by low energy hadronisation and is a topic of strong interest for understanding QCD [3].

There are three different mechanisms of heavy flavour production in a jet system. Heavy flavour quark pair creation gives an insight into perturbative QCD with massive quarks. Heavy flavour quark excitation is sensitive to the heavy-quark distribution in the proton. It produces mainly flavour-asymmetric pairs. Finally, the gluon splitting mechanism is sensitive to non-perturbative QCD dynamics. It also contributes to the mixed flavour jet pair states, but in this case the heavy flavour jet usually contains two heavy quarks. The analysis aimed to measure fractions of the six combinations of dijet events: $f_{B B}, f_{C C}, f_{U U}, f_{B U}, f_{C U}, f_{B C}$, where $B, C$ and $U$ stand for bottom jets, charm jets and jets formed by light quarks or gluons. Jet flavour was identified using templates derived from kinematics of secondary vertex in a jet. No explicit jet flavour tagging was done in this measurement. Jets in an event were reconstructed using the anti- $k_{t}$ algorithm [6] with a distance parameter of $R=0.4$. Jets with $p_{\mathrm{T}}>30 \mathrm{GeV}$ and rapidity $|y|<2.1$ were considered.

Fake vertices might be found in jets which are later classified as light jets and the fake vertex probability is an important parameter which must be the same in data and in Monte Carlo (MC), otherwise all other results cannot be trusted. Figure 1 (left) shows good agreement between data and $\mathrm{MC}$ for fake vertex probability in light jets. Jets with one or two heavy quarks in a jet were considered separately in the analysis and it was found that the $2 b$-jet admixture was higher than predicted by PYTHIA especially at high jet $p_{\mathrm{T}}$ as shown in Figure 1 (middle). The number of leading $\left(N_{b}^{L}\right)$ and subleading $\left(N_{b}^{S L}\right)$ bottom jets was also extracted separately. It was found that the bottom dijet asymmetry $A_{b}=N_{b}^{S L} / N_{b}^{L}-1$ is better described by POWHEG+PYTHIA than by PYTHIA only, see Figure 1 (right).
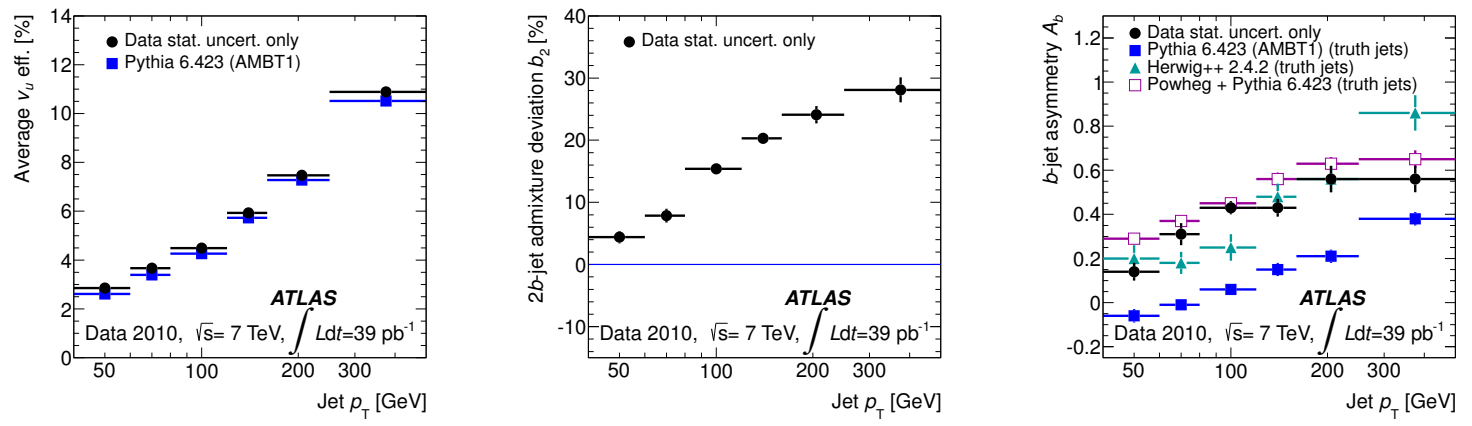

Figure 1: Data fit results for the average fake vertex probability in light jets $v_{u}$ (left), $2 b$-jet admixture deviation $b_{2}$ (middle) and bottom dijet asymmetry $A_{b}$ (right). Only statistical uncertainties are shown. 
Final unfolded flavour compositions for all quark combinations are shown in Figure 2. Most of the distributions are in agreement with leading-order (LO) and next-to-leading order (NLO) calculations except for the bottom+light jet fraction, which is measured to be higher than any of the predictions at $p_{\mathrm{T}}>100 \mathrm{GeV}$.
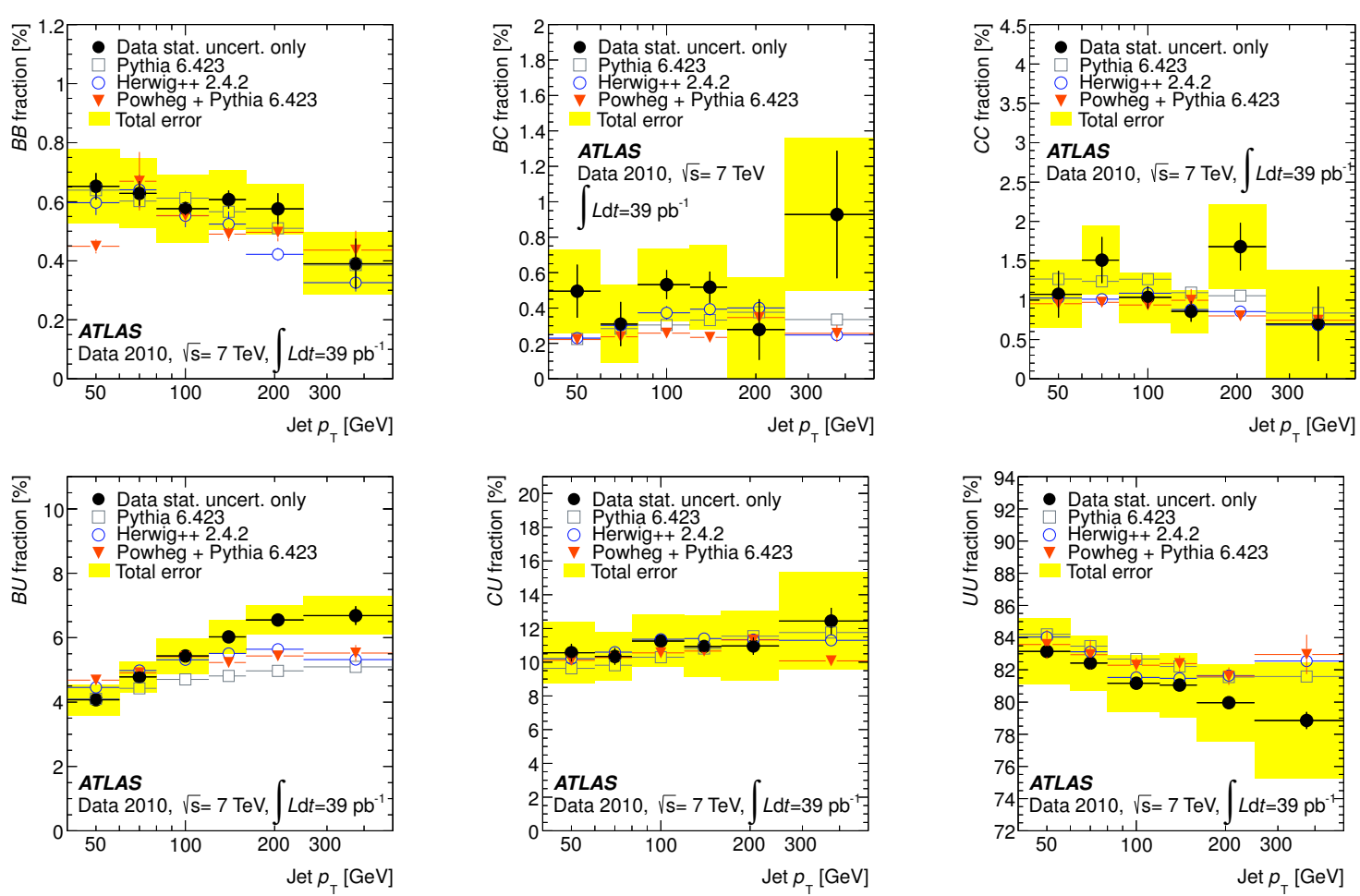

Figure 2: The measured dijet flavour composition for each leading jet $p_{\mathrm{T}}$ bin compared with MC predictions from PYTHIA, HERWIG++ and POWHEG+PYTHIA.

\section{Multi-jet production ratios}

Study of multi-jet events provides valuable insight into theory of strong interactions [4]. The inclusive three-jet to two-jet cross-section ratio is sensitive to the strong coupling constant $\alpha_{s}$ and in such a ratio many systematic uncertainties are cancelled out.

Two possible ratios can be built. The first ratio depends on leading jet $p_{\mathrm{T}}$ :

$$
R_{3 / 2}\left(p_{\mathrm{T}}^{\text {lead }}\right)=\frac{d \sigma_{N_{\text {jets } \geq 3}}}{d p_{\mathrm{T}}^{\text {lead }}} / \frac{d \sigma_{N_{\text {jets }} \geq 2}}{d p_{\mathrm{T}}^{\text {lead }}}
$$

It represents the probability of two-jet events to have a third jet and therefore it is directly proportional to $\alpha_{s}$. The second ratio, which has comparable sensitivity, is obtained by dividing the jet $p_{\mathrm{T}}$ distributions for events with at least three jets to the distribution with at least two jets:

$$
N_{3 / 2}\left(p_{\mathrm{T}}^{(\text {all jets })}\right)=\sum_{i}^{N_{\mathrm{jets}}} \frac{d \sigma_{N_{\mathrm{jets}} \geq 3}}{d p_{\mathrm{T}}^{i}} / \sum_{i}^{N_{\mathrm{jets}}} \frac{d \sigma_{N_{\mathrm{jets}} \geq 2}}{d p_{\mathrm{T}}^{i}}
$$


Jets in this analysis were defined using the anti- $k_{t}$ algorithm with a distance parameter of $R=0.6$. Jets with $p_{\mathrm{T}}>40 \mathrm{GeV}$ and $|y|<2.8$ were considered. In addition, the leading jet was required to have $p_{\mathrm{T}}^{\text {lead }}>60 \mathrm{GeV}$.
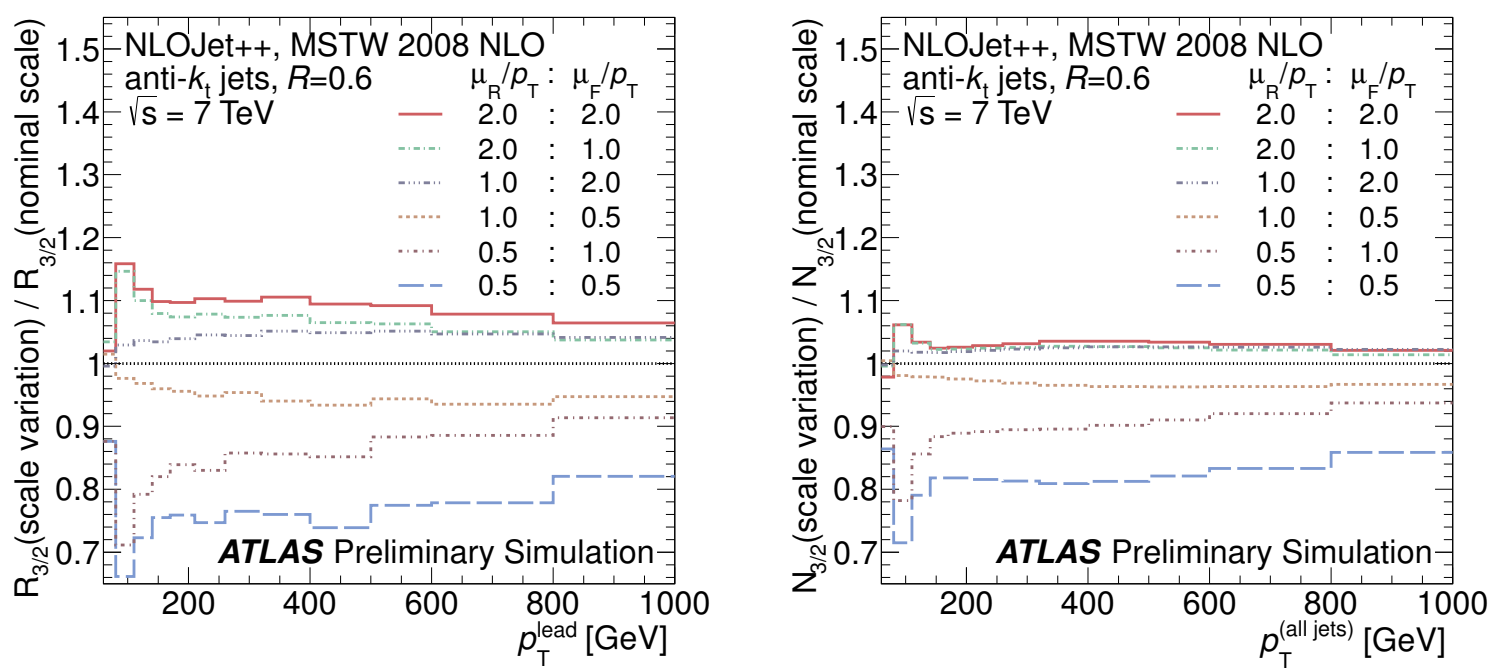

Figure 3: Scale sensitivity of the theoretical predictions for the quantity $R_{3 / 2}$ on the left and $N_{3 / 2}$ on the right, computed at $\alpha_{s}\left(M_{Z}\right)=0.120$. All jets are built from partons using the anti- $k_{t}$ algorithm.

In this measurement $\alpha_{s}$ was determined from comparison to the theoretical predictions using fixed order NLO perturbative QCD calculations with non-perturbative corrections. For $R_{3 / 2}$ predictions renormalisation and factorisation scales were set to the leading jet $p_{\mathrm{T}}\left(\mu_{\mathrm{R}}=\mu_{\mathrm{F}}=p_{\mathrm{T}}^{\text {lead }}\right)$, while for $N_{3 / 2}$ scales were set to $p_{\mathrm{T}}$ of each jet. A detailed study of the behaviour of fixed-order perturbative QCD calculations for different values of $\mu_{\mathrm{R}}$ and $\mu_{\mathrm{F}}$ scales (Figure 3) showed that $N_{3 / 2}$ is more stable against the choice of the scale and therefore it was used for the $\alpha_{s}$ extraction.

The $\alpha_{s}$ at $M_{Z}$ scale was extracted by comparison to NLOJet++ predictions made with $0.110<$ $\alpha_{s}\left(M_{Z}\right)<0.130$ (see Figure 4). A least-squares fit to data was performed, minimising $\chi^{2}$ with respect to $\alpha_{s}\left(M_{Z}\right)$. The fit was done over six $p_{\mathrm{T}}$ bins in the range $[210,800] \mathrm{GeV}$ simultaneously. Correlated systematic uncertainties were included as nuisance parameters and theoretical uncertainties were estimated by altering theoretical predictions. The fit result $\alpha_{s}\left(M_{Z}\right)=0.111 \pm$ $0.006(\exp )_{-0.0003}^{+0.016}$ (theory) is in agreement with the PDG value $0.1184 \pm 0.0007$ within uncertainties. The most important contribution to the experimental uncertainty is the jet energy scale while for the theoretical uncertainty the scale uncertainty dominates.

The energy scale dependence of $\alpha_{s}$ was determined by extracting $\alpha_{s}\left(M_{Z}\right)$ from each $p_{\mathrm{T}}$ bin individually. These $\alpha_{s}\left(M_{Z}\right)$ were transformed to $\alpha_{s}$ at the average $p_{\mathrm{T}}$ value in each bin using the two-loop approximate solution to the Renormalisation Group Equation (RGE). The observed energy scale dependence of $\alpha_{s}$ is in good agreement with previous measurements and it is consistent with the RGE preditions up to an energy scale of $800 \mathrm{GeV}$ (see Figure 5).

\section{4. $k_{T}$ splitting scales in $W+$ jets events}

Measurement of splitting scales in the $k_{\mathrm{T}}$ clustering algorithm using jets from $W+$ jets events [5] 


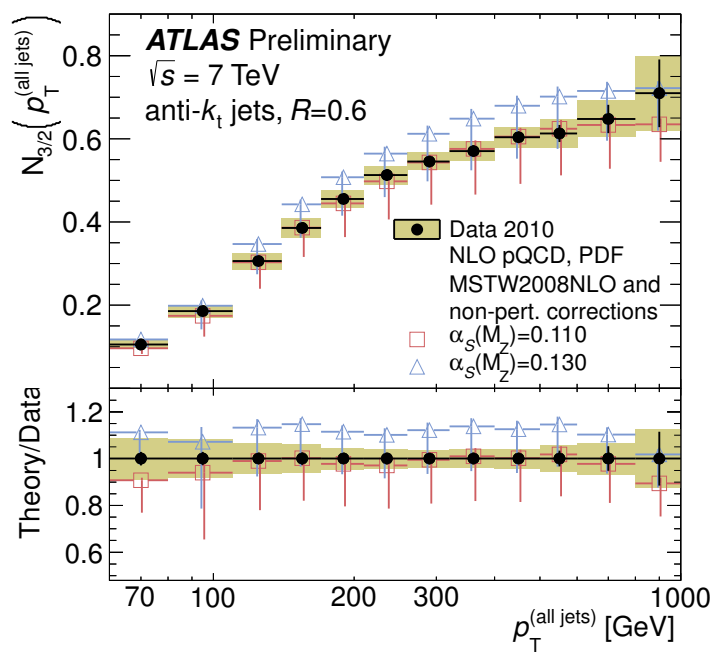

Figure 4: Measurement of $N_{3 / 2}$, at particle level, as a function of $p_{\mathrm{T}}^{\text {(all jets) }}$. NLO pQCD theoretical predictions, corrected for non-perturbative effects, are also shown for a value of $\alpha_{s}\left(M_{Z}\right)$ of 0.110 and 0.130 .

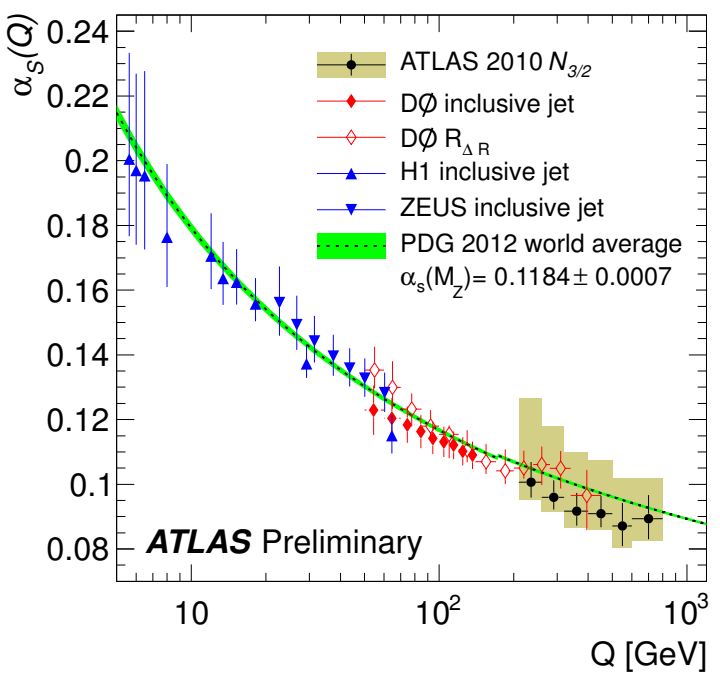

Figure 5: Strong coupling constant $\alpha_{s}$ extracted from ATLAS, D0, ZEUS and $\mathrm{H} 1$ as a function of the renormalisation scale $Q$.

can probe QCD evolution, provides useful test of LO and NLO QCD Monte Carlo generators and analytical calculations.

Splitting scale $\sqrt{d_{k}}$ is defined as a minimal distance $\sqrt{d_{\min }}$ between momenta calculated by the $k_{\mathrm{T}}$ algorithm when going from $k+1$ to $k$ constituents. These momenta can be determined using energy deposits (clusters) in the calorimeter at the detector level, or hadrons at the particle level in Monte Carlo simulation. For example, in two-jet events $\sqrt{d_{0}}$ is the transverse $p_{\mathrm{T}}$ of the leading jet, $\sqrt{d_{1}}$ is the transverse $p_{\mathrm{T}}$ of the subleading jet, and $\sqrt{d_{2}}$ is the distance between second and third momenta which form one jet.

Events with $W \rightarrow e v$ and $W \rightarrow \mu v$ decays were selected for this analysis and all calorimeter clusters within a pseudorapidity $|\eta|<4.9$ were used for $k_{\mathrm{T}}$ algorithm with a distance parameter of $R=0.6$. Seven observables were measured: four splitting scales for $0 \leq k \leq 3$ in which clear separation between soft and hard regions is visible and three ratios of subsequent scales $\sqrt{d_{k+1}} / \sqrt{d_{k}}$ for $0 \leq k \leq 2$. In the ratio, systematic errors are cancelled to some extend. A cut $\sqrt{d_{k}}>20 \mathrm{GeV}$ was applied in the ratio to avoid domination by non-perturbative effects. The ratio $\sqrt{d_{k+1}} / \sqrt{d_{k}} \rightarrow 1$ is of particular interest as it probes events with subsequent emissions at similar scale.

Detector-level comparison for splitting scales $\sqrt{d_{0}}$ and $\sqrt{d_{3}}$ are shown in Figure 6. In general, a good agreement between ALPGEN+HERWIG MC and data is observed. The most visible difference between $\sqrt{d_{0}}$ and $\sqrt{d_{3}}$ distributions is the larger $t \bar{t}$ background at high $\sqrt{d_{3}}$, since it is sensitive to four-jet production. The same distributions corrected for detector resolution and pile-up effects are shown in Figure 7 and they are compared with different MC generators. ALPGEN+HERWIG works very well in the hard tail, other generators underestimate the results from data (even in $\sqrt{d_{0}}$ ). HERWIG-based generators are best in soft (resummation) region and an excess of SHERPA and MC@NLO in the intermediate region is clearly visible. Results for ratio 

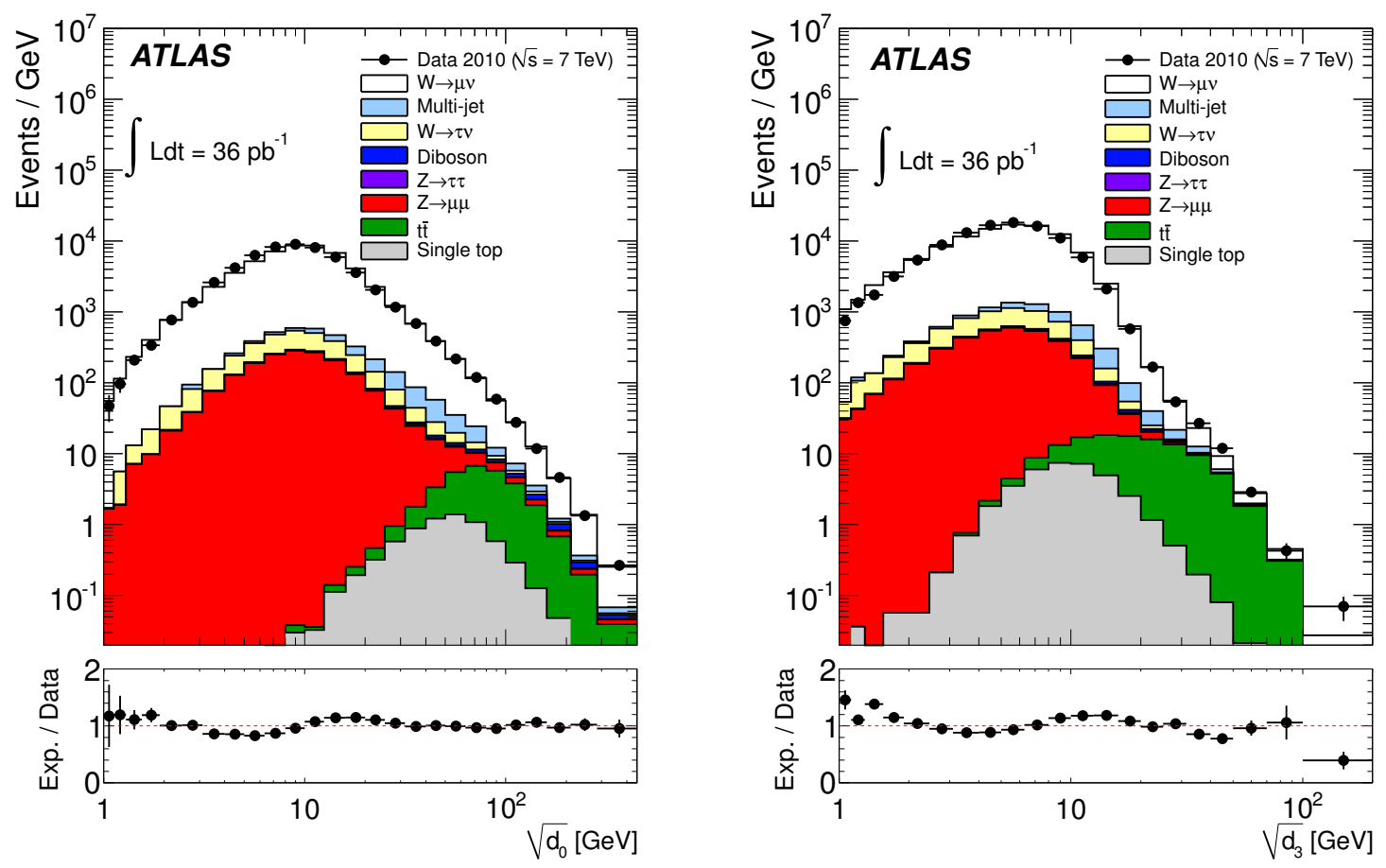

Figure 6: Uncorrected splitting scale $\sqrt{d_{0}}$ (left) and $\sqrt{d_{3}}$ (right) for events passing the $W \rightarrow \mu v$ selection requirements. The distributions from the data (markers) are compared with the predicted signal from the MC simulation, provided by ALPGEN and normalised to the next-to-next-to-leading-order (NNLO) prediction.
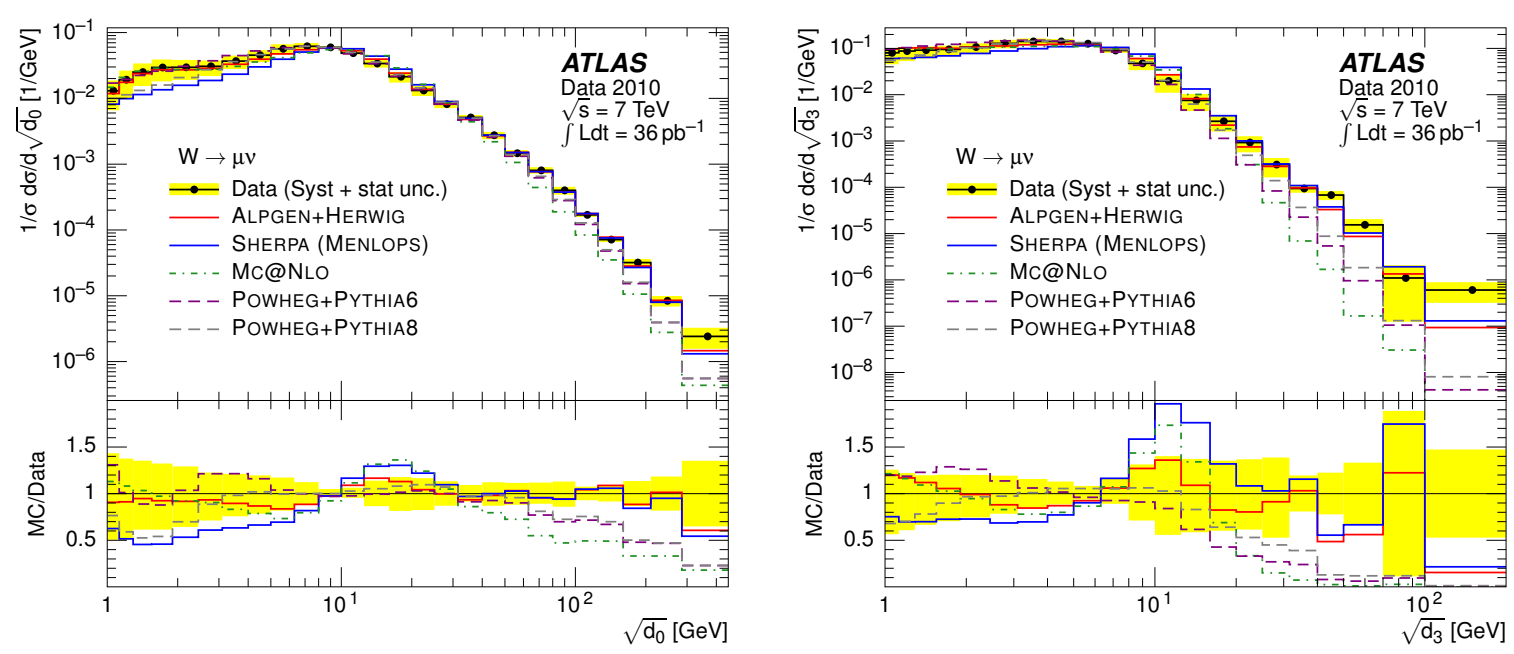

Figure 7: Distributions of $\sqrt{d_{0}}$ (left) and $\sqrt{d_{3}}$ (right) in the $W \rightarrow \mu \nu$ channel, shown at particle level. The data (markers) are compared to the predictions from various MC generators, and the shaded bands represent the quadratic sum of systematic and statistical uncertainties on each bin.

observables are shown in Figure 8. HERWIG-based generators provide good description of leading ratio, while POWHEG+PYTHIA6 is not so good. At higher ratios most generators are just outside uncertainty. This information can be used for tuning the MC generators in the future. 

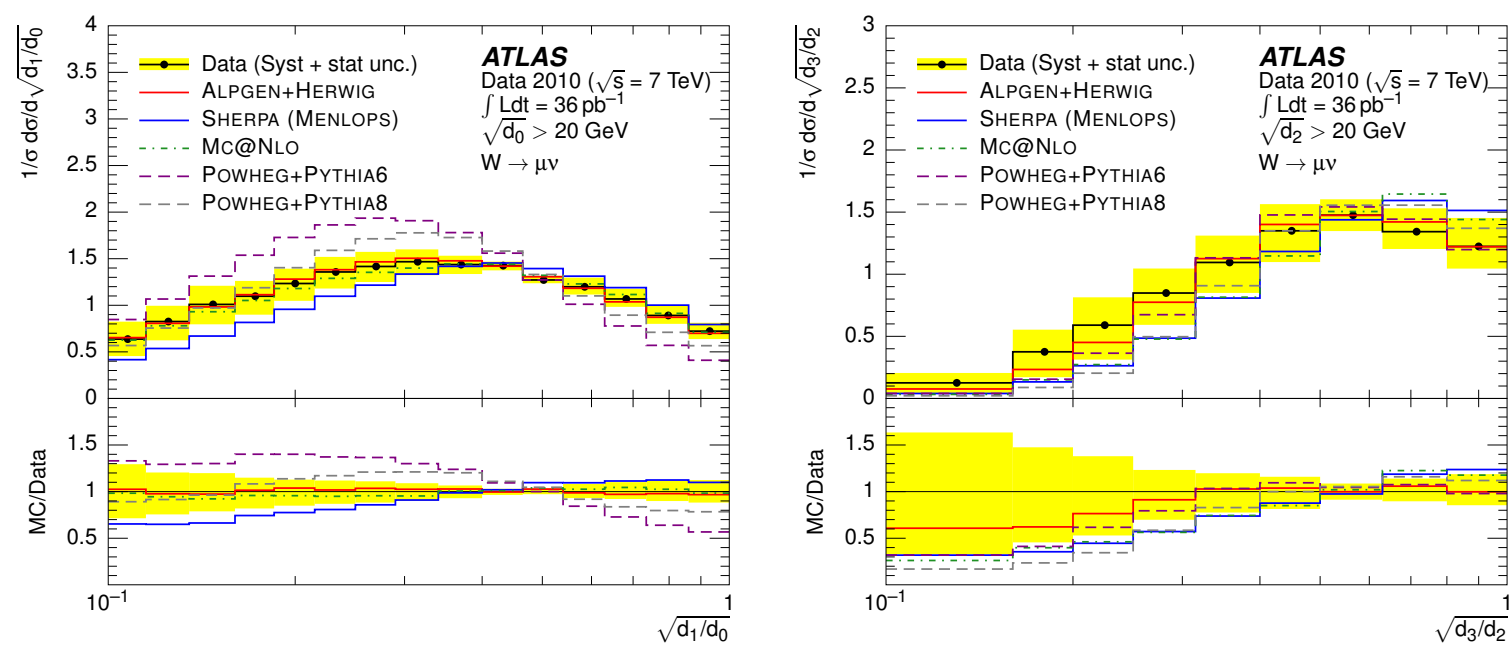

Figure 8: Distributions of $\sqrt{d_{1} / d_{0}}$ on the left and $\sqrt{d_{3} / d_{2}}$ on the right in the $W \rightarrow \mu v$ channel, shown at particle level. The data (markers) are compared to the predictions from various MC generators, and the shaded bands represent the quadratic sum of systematic and statistical uncertainties on each bin.

\section{Conclusions}

An analysis of jet flavour composition of dijet events is an excellent tool to study perturbative QCD and to validate MC generators. Bottom+light flavour composition is found to be larger than the NLO or LO predictions. Other flavour compositions are reproduced by the predictions.

The $N_{3 / 2}$ observable in the analysis of multi-jet events provides a direct measurement of the strong coupling constant. The value of $\alpha_{s}\left(M_{Z}\right)$ derived with a global fit is in agreement with the PDG value. The measurement of $\alpha_{s}$ energy scale dependence is extended up to a scale of $800 \mathrm{GeV}$.

A measurement of the $k_{\mathrm{T}}$ splitting scales in $W+$ jets events improves the theoretical modelling of QCD effects and provides useful test of LO and NLO QCD Monte Carlo generators. Leading order multi-leg predictions perform better than NLO generators especially in hard tails. Significant differences are found in the soft region.

\section{References}

[1] ATLAS Collaboration, The ATLAS Experiment at the CERN Large Hadron Collider, JINST 3 (2008) S08003.

[2] L. Evans and P. Bryant (editors), LHC Machine, JINST 3 (2008) S08001.

[3] ATLAS Collaboration, Measurement of the flavour composition of dijet events in pp collisions at $\sqrt{s}=7 \mathrm{TeV}$ with the ATLAS detector, Eur. Phys. J. C 73(2013) 2301.

[4] ATLAS Collaboration, Measurement of multi-jet cross-section ratios and determination of the strong coupling constant in proton-proton collisions at $\sqrt{s}=7 \mathrm{TeV}$ with the ATLAS detector, ATLAS CONF-2013-041, https://cds.cern.ch/record/1543225.

[5] ATLAS Collaboration, Measurement of $k_{\mathrm{T}}$ splitting scales in $W \rightarrow l v$ events at $\sqrt{s}=7 \mathrm{TeV}$ with the ATLAS detector, Eur. Phys. J. C 735 (2013) 2432.

[6] M. Cacciari, G. Salam and G. Soyez, The anti- $k_{t}$ jet clustering algorithm, JHEP 04 (2008) 063. 